\title{
Extended dynamical symmetries of Landau levels in higher dimensions
}

\author{
S. Kürkçüoğlu, ${ }^{a}$ G. Ünal ${ }^{b}$ and i. Yurdușen ${ }^{c}$ \\ ${ }^{a}$ Department of Physics, Middle East Technical University, \\ Dumlupinar Boulevard, 06800, Ankara, Turkey \\ ${ }^{b}$ Department of Physics, İmir Institute of Technology, IZTECH, \\ 35430, İmir, Turkey \\ ${ }^{c}$ Department of Mathematics, Hacettepe University, \\ 06800, Beytepe, Ankara, Turkey \\ E-mail: kseckin@metu.edu.tr, gonulunal@iyte.edu.tr, \\ yurdusen@hacettepe.edu.tr
}

ABSTRACT: Continuum models for time-reversal (TR) invariant topological insulators (TIs) in $d \geq 3$ dimensions are provided by harmonic oscillators coupled to certain $\mathrm{SO}(d)$ gauge fields. These models are equivalent to the presence of spin-orbit (SO) interaction in the oscillator Hamiltonians at a critical coupling strength (equivalent to the harmonic oscillator frequency) and leads to flat Landau Level (LL) spectra and therefore to infinite degeneracy of either the positive or the negative helicity states depending on the sign of the SO coupling. Generalizing the results of [1] to $d \geq 4$, we construct vector operators commuting with these Hamiltonians and show that $\mathrm{SO}(d, 2)$ emerges as the non-compact extended dynamical symmetry. Focusing on the model in four dimensions, we demonstrate that the infinite degeneracy of the flat spectra can be fully explained in terms of the discrete unitary representations of $\mathrm{SO}(4,2)$, i.e. the doubletons. The degeneracy in the opposite helicity branch is finite, but can still be explained exploiting the complex conjugate doubleton representations. Subsequently, the analysis is generalized to $d$-dimensions, distinguishing the cases of odd and even $d$. We also determine the spectrum generating algebra in these models and briefly comment on the algebraic organization of the LL states w.r.t. an underlying "deformed" AdS geometry as well as on the organization of the surface states under open boundary conditions in view of our results.

KEYwords: Gauge Symmetry, Topological States of Matter

ArXiv EPRINT: 1909.10446 


\section{Contents}

1 Introduction 1

2 The model for four-dimensional LLs 3

2.1 Basics and preliminary remarks 3

2.2 Extended dynamical symmetries 5

3 Discrete UIRs of $\mathrm{SO}(4,2)$ and the degenerate LL spectrum 7

4 Generalization to $d$-dimensions $\quad 11$

$\begin{array}{lll}5 & \text { Spectrum generating algebra } & 14\end{array}$

$\begin{array}{lll}6 & \text { Discussion and conclusions } & 15\end{array}$

$\begin{array}{ll}\text { A } s o(4,2) & \text { Lie algebra in the Cartan-Weyl basis } \\ \end{array}$

$\begin{array}{ll}\text { B Details of the construction of doubletons } & 18\end{array}$

\section{Introduction}

There is ongoing intense interest in gaining further theoretical insights from new and diverse perspectives on the topological phases of matter discovered in the past decade or so. This is reflected in the vast and diverse literature on the subject [1-18]. Integer and fractional quantum Hall $(\mathrm{QH})$ states [19-25], which were discovered in early 80's, may be interpreted as early examples, which carry distinct topological numbers distinguishing them from the ordinary states of matter. Higher dimensional generalizations QHE are also well-known [27-31].

More recent interest in the field is driven by the discovery of the new topological phases of matter in two and subsequently in three and higher dimensions, which respect the TR symmetry. In two-dimensions, Topological insulators (TIs) with TR invariance are investigated using both Bloch wave band structure $[2,26]$, as well as via introducing continuum models [3, 4]. For instance, quantum spin Hall effect (QSHE) [3] can be understood as consisting of two integer QH states with opposite chirality, for which the charge Hall conductance vanishes, while the spin Hall conductance is quantized (in units of $\frac{e}{2 \pi}$ ).

New phases of matter with non-trivial topology in three and higher dimensions are also being investigated rigorously from several different directions in the recent past $[5-10,16,17]$. Li and $\mathrm{Wu}[15]$ introduced an interesting continuum model for three and higher dimensional TR invariant TIs. The model proposed by these authors is described by Hamiltonians, in which the charged spin $1 / 2$ particles are non-minimally coupled to 
$\mathrm{SU}(2) \simeq \mathrm{SO}(3)$ and $\mathrm{SO}(d)$, Aharanov-Casher type non-abelian gauge fields in three and $d$-dimensions, respectively. These Hamiltonians can also be expressed as that of three and $d$-dimensional harmonic oscillators with a spin-orbit (SO) term at a critical coupling strength, matching the frequency of the harmonic oscillator and has the property that, depending on the sign of the SO term, either the positive or the negative helicity component exhibits flat spectra. The latter is a characteristic of the Landau levels, and therefore the models proposed in [15] may be taken as the generalization of the TR invariant LLs of QSHE [3] to flat higher-dimensional spaces. Authors of [15] have shown that, these models posses gapless helical Dirac surface states, which are robust against the TR invariant perturbations and therefore allow for the interpretation of the models as TR preserving TIs with a $\mathbb{Z}_{2}$ topological invariant.

In [1], properties and structure of the wave functions of the three dimensional TI system are explored from an algebraic perspective and an underlying non-compact extended dynamical symmetry group that completely accounts for both the infinite degeneracy of the LLs in the positive helicity and the finite degeneracy in the negative helicity component is determined. In practice, authors of [1] reveal two vector operators involving the total angular momentum (orbital and spin) and commuting with the Hamiltonian. Appropriately scaled form of these vector operators on the eigenstates of the Hamiltonian and the generators of the total angular momentum, span the noncompact Lie algebra so(3,2). The Dirac Singleton $[32,33$ ] is a well-known unitary irreducible representation (UIR) of $s o(3,2)$ and plays the central role in explaining the aforementioned infinite and finite degeneracies in the spectrum of this model. It may be noted that, knowledge on the algebraic structure of the TI wave functions could be very useful, since it could allow for a deeper understanding of $i$. the underlying geometric features and $i i$. the algebraic organization of the surface states. To elaborate on the first item, it is speculated in [1] that the underlying geometry of LLs in three dimensions could be related to some radially deformed form of $A d S_{4}$, rather than the flat space, since the extended symmetry group can be thought as a "deformed" $\mathrm{SO}(3,2)$, recalling that $\mathrm{SO}(3,2)$ is the exact isometry group of $A d S_{4}$, while for the second we may recall the $W_{\infty}$ symmetry encountered in certain QH phases, which accounts for the incompressibility of the QH droplet and algebra of the edge states [34-37], whose generalization to TR invariant TIs would be very interesting.

In the present work, we extend the results of [1] to four and subsequently to $d$ dimensional models. Introducing the appropriate vector operators commuting with the Hamiltonian and obtaining their suitably scaled form acting on the energy eigenkets, the extended dynamical symmetry group in four dimensions is identified as $\mathrm{SO}(4,2)$. We show in full detail how the infinite degeneracy of the energy spectrum in the positive helicity branch can be explained in terms of the discrete UIRs of $\mathrm{SO}(4,2)$, which are also known as the doubletons [38-40]. The finite degeneracy in the negative branch is also understood using the complex conjugate doubleton representations. In section 4 , we give the generalization of our analysis to the models in $d$-dimensions and reveal that the extended symmetry group is $\mathrm{SO}(d, 2)$, while the degeneracies are explained using the discrete series UIRs of $\mathrm{SO}(d, 2)$, which essentially generalize the singleton and the doubleton representations in $d=3$ and $d=4$ to higher odd and even dimensions, respectively [41,42]. We conclude by 
making some brief remarks regarding the algebraic organization of the LL states w.r.t. an underlying "deformed" AdS geometry and on the organization of the surface states under open boundary conditions.

\section{The model for four-dimensional LLs}

\subsection{Basics and preliminary remarks}

We may launch our discussion starting with the Hamiltonian of a four-dimensional $(4 D)$ harmonic oscillator coupled to an Aharanov-Casher type $\mathrm{SO}(4)$ gauge field $G_{a}=2 m \omega r_{b} S_{a b}$ in the form [15]

$$
H=\frac{1}{2 m}\left(p_{a}-G_{a}\right)^{2}-m \omega^{2} r_{a}^{2},
$$

which may be expressed as the Hamiltonian for a simple harmonic oscillator (SHO) with the spin-orbit (SO) term at the coupling strength $\omega$ matching the SHO frequency as

$$
H=\frac{p_{a}^{2}}{2 m}+\frac{1}{2} m \omega^{2} r_{a}^{2}-\omega \sum_{a<b=1}^{4} L_{a b} \Gamma_{a b} .
$$

In this expression $L_{a b}:=r_{a} p_{b}-r_{b} p_{a},(a, b=1, \cdots, 4)$ are the orbital angular momentum operators, while $\Gamma_{a b}$ are proportional to the spin operator $S_{a b}$ in 4-dimensions, as will be explicitly defined in what follows. Setting $\hbar=1$, we may write the momentum operator as $p_{a}=-i \partial_{a}$. In terms of the representation theory of $\mathrm{SO}(4), L_{a b}$ carries the $(l, 0)$ irreducible representation (IRR) of $\mathrm{SO}(4)$, while $S_{a b}$ carries the direct sum representation $(1 / 2,1 / 2) \oplus$ $(1 / 2,-1 / 2)$ (IRRs are given in the highest weight i.e. the Gelfand-Zeitlin notation). To be more concrete, let us introduce the 4-dimensional Euclidean $\gamma$-matrices, $\gamma_{a},(a=1, \cdots, 4)$ with the anti-commutation relations $\left\{\gamma_{a}, \gamma_{b}\right\}=2 \delta_{a b}$. We may choose them to be of the form

$$
\gamma_{i}=\left(\begin{array}{cc}
0 & -i \sigma_{i} \\
i \sigma_{i} & 0
\end{array}\right), \quad \gamma_{4}=\left(\begin{array}{ll}
0 & 1 \\
1 & 0
\end{array}\right), \quad \gamma_{5}=\gamma_{1} \gamma_{2} \gamma_{3} \gamma_{4}=\left(\begin{array}{cc}
1 & 0 \\
0 & -1
\end{array}\right),
$$

where $\sigma_{i},(i=1,2,3)$ are the usual Pauli matrices.

Spin operator, $S_{a b}$, may be expressed as

$$
S_{a b}:=\frac{1}{2} \Gamma_{a b}:=-\frac{i}{4}\left[\gamma_{a}, \gamma_{b}\right], \quad S_{a b}=\left(\begin{array}{cc}
S_{a b}^{+} & 0 \\
0 & S_{a b}^{-}
\end{array}\right), \quad S_{a b}^{ \pm}=\left(S_{i j}, \mp \frac{1}{2} \sigma_{i}\right)=\left(\frac{1}{2} \varepsilon_{i j k} \sigma_{k}, \mp \frac{1}{2} \sigma_{i}\right) .
$$

Total angular momentum is given as $J_{a b}=L_{a b}+S_{a b}$ and has the IRR content given by the decomposition of the product $(l, 0) \otimes[(1 / 2,1 / 2) \oplus(1 / 2,-1 / 2)]$ as

$$
\left(l+\frac{1}{2}, \frac{1}{2}\right) \oplus\left(l-\frac{1}{2}, \frac{1}{2}\right) \oplus\left(l+\frac{1}{2},-\frac{1}{2}\right) \oplus\left(l-\frac{1}{2},-\frac{1}{2}\right) .
$$

$\mathrm{SO}(4)$ commutation relations are given in terms of generic generators $M_{a b}$ as

$$
\left[M_{a b}, M_{c d}\right]=i\left(\delta_{a c} M_{b d}+\delta_{b d} M_{a c}-\delta_{a d} M_{b c}-\delta_{b c} M_{a d}\right) .
$$

$L_{a b}, S_{a b}$ and $J_{a b}$ satisfy (2.6). 
The Hamiltonian commutes with the total angular momentum operator $J_{a b}$. Its spectrum and eigenfunctions are given in [15]. We briefly present some details in order to be self-contained and prepare for the developments that follow. Spectrum of the pure $4 D$ SHO is given as $E_{4 D, S H O}=\omega(2 n+\ell+2)$ and the corresponding energy eigenfunctions are of the form $\Psi(r, \theta, \phi, \psi)=R_{n \ell}(r) Y_{m_{L} m_{R}}^{l}(\theta, \phi, \psi)$, where $R_{n \ell}(r)=r^{l} e^{-\frac{1}{2} m \omega r^{2}} F\left(-n, l+2, m \omega r^{2}\right)$ with $n \in \mathbb{Z}_{+}$is the radial wave function and $Y_{m_{L} m_{R}}^{\ell}(\theta, \phi, \psi)$ are the spherical harmonics in four dimensions.

Eigenvalues of the SO term can easily be worked out using the eigenvalues of the Casimir operators for the IRRs appearing in (2.5). We have

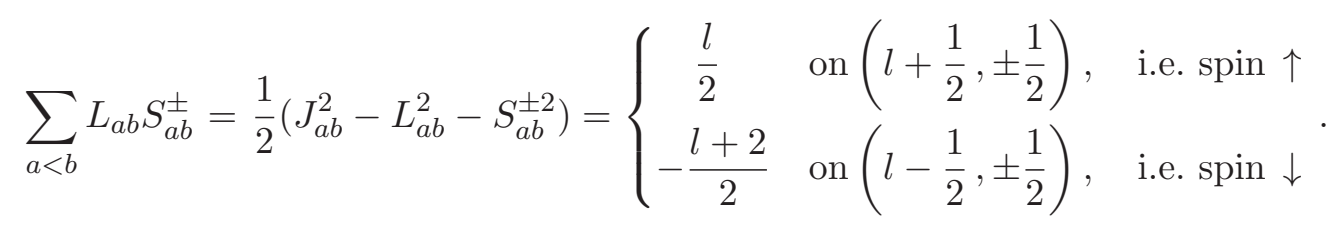

Spectrum of the Hamiltonian in (2.2) then follows as

$$
E=\left\{\begin{array}{ll}
2 \omega(n+1), & \text { spin } \uparrow \\
2 \omega(n+l+2), & \text { spin } \downarrow
\end{array},\right.
$$

from which we observe that the spin up (positive SO branch) part has flat spectrum, i.e. it is independent of the orbital angular momentum $l$, and leads to an infinite degeneracy at each energy level. Spin down (negative SO branch) part of the spectrum is also degenerate, but not infinitely so. In the ensuing sections our main focus will be explaining the reason underlying this degeneracy. It is useful to note that the infinite degeneracy of the positive SO branch is a direct consequence of the critical SO coupling strength which matches with the SHO frequency $\omega$; in particular, changing the sign of the SO term in the Hamiltonian would flip the spectrum of the positive and negative SO branches, making the latter infinitely degenerate instead. Corresponding wave functions are $R_{n l}(r) \mathcal{Y}_{l ; m_{L} m_{R}}^{l \pm \frac{1}{2}}(\theta, \phi, \psi)$ where $R_{n l}(r)$ is the same as before, while $\mathcal{Y}_{l ; m_{L} m_{R}}^{l \pm \frac{1}{2}}(\theta, \phi, \psi)$ are the spin spherical harmonics in four dimensions.

In analogy with the discussion of [1] in three dimensions, we find it useful to introduce the operator

$$
A=\sum_{a<b} L_{a b} \Gamma_{a b}+\frac{3}{2}
$$

Using equation (2.7) eigenvalues of $A$ can be simply written as $l^{\prime}:=l+\frac{3}{2}$ for spin up and $l^{\prime}:=-l-\frac{1}{2}$, for spin down, respectively. Thus, we have $l^{\prime}= \pm \frac{3}{2}, \pm \frac{5}{2}, \cdots$. Evidently, $A$ commutes with the Hamiltonian and therefore its eigenvalues $l^{\prime}$ can be used in labeling the energy eigenstates. Since $\mathrm{SO}(4) \simeq \mathrm{SU}(2) \times \mathrm{SU}(2)$, we can introduce $\mathrm{SU}(2)$-left and $\mathrm{SU}(2)$-right generators for the total angular momentum $J_{a b}$ as

$$
L_{i}=\frac{1}{2}\left(\frac{1}{2} \varepsilon_{i j k} J_{j k}+J_{i 4}\right), \quad R_{i}=\frac{1}{2}\left(\frac{1}{2} \varepsilon_{i j k} J_{j k}-J_{i 4}\right)
$$


with the commutation relations

$$
\left[L_{i}, L_{j}\right]=i \varepsilon_{i j k} L_{k}, \quad\left[R_{i}, R_{j}\right]=i \varepsilon_{i j k} R_{k}, \quad\left[L_{a}, R_{b}\right]=0 .
$$

Conventionally, generators of the Cartan subgroup of $\mathrm{SO}(4)$ are taken as $\left(J_{12}, J_{34}\right)$, while for $\mathrm{SU}(2) \times \mathrm{SU}(2)$ they are taken as

$$
\left(L_{3}, R_{3}\right)=\left(\frac{1}{2}\left(J_{12}+J_{34}\right), \frac{1}{2}\left(J_{12}-J_{34}\right)\right) .
$$

From (2.5) and (2.12) we see that the fundamental representations $(1 / 2,1 / 2)$ and $(1 / 2,-1 / 2)$ correspond respectively to $\left(L_{i}, R_{i}\right) \equiv\left(0, \frac{\sigma_{i}}{2}\right)$ and $\left(L_{i}, R_{i}\right) \equiv\left(\frac{\sigma_{i}}{2}, 0\right)$. In the $\mathrm{SU}(2) \times \mathrm{SU}(2)$ irreducible representation notation, $\left(j_{1}, j_{2}\right)$, these are labeled as $(0,1 / 2)$ and $(1 / 2,0)$, respectively.

We choose to label the eigenstates of the Hamiltonian in terms of the principal quantum number $n$ and the eigenvalues $l^{\prime}, m_{L}, m_{R}$ of $A, L_{3}$ and $R_{3}$, and denote, in the Dirac notation, these states as $\left|n, l^{\prime}, m_{L}, m_{R}\right\rangle$ with

$$
\begin{aligned}
A\left|n, l^{\prime}, m_{L}, m_{R}\right\rangle & =l^{\prime}\left|n, l^{\prime}, m_{L}, m_{R}\right\rangle, \\
L_{3}\left|n, l^{\prime}, m_{L}, m_{R}\right\rangle & =m_{L}\left|n, l^{\prime}, m_{L}, m_{R}\right\rangle, \\
R_{3}\left|n, l^{\prime}, m_{L}, m_{R}\right\rangle & =m_{R}\left|n, l^{\prime}, m_{L}, m_{R}\right\rangle .
\end{aligned}
$$

In terms of the $\mathrm{SU}(2) \otimes \mathrm{SU}(2)$ representation labels the direct sum representation in (2.5) reads

$$
\left(\frac{l+1}{2}, \frac{l}{2}\right) \oplus\left(\frac{l}{2}, \frac{l-1}{2}\right) \oplus\left(\frac{l}{2}, \frac{l+1}{2}\right) \oplus\left(\frac{l-1}{2}, \frac{l}{2}\right)
$$

From this, we immediately infer that $\left|m_{L}\right| \leq \frac{l+1}{2}$ and $\left|m_{R}\right| \leq \frac{l}{2}$, and $\left|m_{L}\right| \leq \frac{l}{2}$ and $\left|m_{R}\right| \leq$ $\frac{l-1}{2}$ respectively, for the right chiral representations (i.e. first two summands in (2.5)). In terms of the eigenvalues of $l^{\prime}$ of $A$, we have the range of eigenvalues for $m_{L}$ and $m_{R}$ expressed as

$$
\left|m_{L}\right| \leq\left(\frac{\left|l^{\prime}\right|}{2}-\frac{1}{4}\right), \quad\left|m_{R}\right| \leq\left(\frac{\left|l^{\prime}\right|}{2}-\frac{3}{4}\right) .
$$

As for the range of values for $m_{L}$ and $m_{R}$ in the left chiral representations, we simply interchange $m_{L}$ and $m_{R}$ in (2.15).

Trading the label $l$ for $l^{\prime}$, we can express the spectrum in (2.8) as

$$
E=\left\{\begin{array}{ll}
2 \omega(n+1), & \text { spin } \uparrow \\
2 \omega\left(n-l^{\prime}+\frac{3}{2}\right), & \text { spin } \downarrow
\end{array} .\right.
$$

\subsection{Extended dynamical symmetries}

In order to understand the infinite and the finite degeneracies of the positive and negative helicity branches of the spectrum (2.16), we will reveal this $4 D$ model has an extended noncompact dynamical symmetry group. To do so, working from now on with $m=1$ and $\omega=$ 
$\frac{1}{2}$, generalizing the approach of [1], we introduce two Hermitian vector operators commuting with the Hamiltonian $H$ and involve, in addition to the coordinates and momenta, the total angular momentum and the SO operator $A$. Explicitly, they are in the form

$$
\begin{aligned}
M_{a} & =\frac{1}{4}\left(r_{a} A+A r_{a}\right)+\frac{1}{2}\left(p_{b} J_{a b}+J_{a b} p_{b}\right), \\
N_{a} & =\frac{1}{2}\left(p_{a} A+A p_{a}\right)-\frac{1}{4}\left(r_{b} J_{a b}+J_{a b} r_{b}\right) .
\end{aligned}
$$

It can be straightforwardly demonstrated that

$$
\left[M_{a}, H\right]=0, \quad\left[N_{a}, H\right]=0,
$$

and $M_{a}$ and $N_{a}$ transform as vectors under the adjoint action of $J_{a b}$ :

$$
\begin{aligned}
a d J_{a b} M_{c} & :=\left[J_{a b}, M_{c}\right]=i \delta_{a c} M_{b}-i \delta_{b c} M_{a}, \\
a d J_{a b} N_{c} & :=\left[J_{a b}, N_{c}\right]=i \delta_{a c} N_{b}-i \delta_{b c} N_{a},
\end{aligned}
$$

by direct calculation. It is also useful to note that the commutators of these vector operators with $A$, take the form

$$
\left[A, M_{a}\right]=-i N_{a}, \quad\left[A, N_{a}\right]=i M_{a} .
$$

A set of long and rather tedious calculations yield the commutation relations for the operators $M_{a}$ and $N_{a}$ as

$$
\begin{aligned}
{\left[M_{a}, M_{b}\right] } & =-2 i J_{c d}\left(\delta_{a c} \delta_{b d}\left(H+\frac{3}{2} A-1\right)+\frac{1}{8} \varepsilon_{a b c d} \gamma_{5}\right) \\
{\left[N_{a}, N_{b}\right] } & =-2 i J_{c d}\left(\delta_{a c} \delta_{b d}\left(H+\frac{3}{2} A-1\right)+\frac{1}{8} \varepsilon_{a b c d} \gamma_{5}\right) \\
{\left[M_{a}, N_{b}\right] } & =2 i \delta_{a b} A\left(H+\frac{3}{2} A-1\right)+i J_{a c} J_{b c}-i \delta_{a b} \sum_{c<d} J_{c d}^{2} \\
& =2 i \delta_{a b}\left(A\left(H+\frac{3}{2} A-1\right)-\frac{1}{2} A^{2}+\frac{3}{8}\right)+i J_{a c} J_{b c} .
\end{aligned}
$$

We have used, $\sum_{c<d} J_{c d}^{2}=A^{2}-\frac{3}{4}$ to express the second line of the last commutator in (2.21). We may form the following linear combinations of $M_{a}$ and $N_{a}$

$$
\begin{aligned}
K_{ \pm}^{1} & :=\frac{1}{\sqrt{2}}\left(M_{1} \pm i M_{2} \mp i N_{1}+N_{2}\right), \\
K_{ \pm}^{2} & :=\frac{1}{\sqrt{2}}\left(M_{1} \mp i M_{2} \mp i N_{1}-N_{2}\right), \\
K_{ \pm}^{3} & :=\frac{1}{\sqrt{2}}\left(M_{3} \pm i M_{4} \mp i N_{3}+N_{4}\right), \\
K_{ \pm}^{4} & :=\frac{1}{\sqrt{2}}\left(M_{3} \mp i M_{4} \mp i N_{3}-N_{4}\right),
\end{aligned}
$$

which fulfill the commutation relations

$$
\begin{array}{lll}
{\left[A, K_{ \pm}^{1}\right]= \pm K_{ \pm}^{1},} & {\left[L_{3}, K_{ \pm}^{1}\right]= \pm \frac{1}{2} K_{ \pm}^{1},} & {\left[R_{3}, K_{ \pm}^{1}\right]= \pm \frac{1}{2} K_{ \pm}^{1},} \\
{\left[A, K_{ \pm}^{2}\right]= \pm K_{ \pm}^{2},} & {\left[L_{3}, K_{ \pm}^{2}\right]=\mp \frac{1}{2} K_{ \pm}^{2},} & {\left[R_{3}, K_{ \pm}^{2}\right]=\mp \frac{1}{2} K_{ \pm}^{2},} \\
{\left[A, K_{ \pm}^{3}\right]= \pm K_{ \pm}^{3},} & {\left[L_{3}, K_{ \pm}^{3}\right]= \pm \frac{1}{2} K_{ \pm}^{3},} & {\left[R_{3}, K_{ \pm}^{3}\right]=\mp \frac{1}{2} K_{ \pm}^{3},} \\
{\left[A, K_{ \pm}^{4}\right]= \pm K_{ \pm}^{4},} & {\left[L_{3}, K_{ \pm}^{4}\right]=\mp \frac{1}{2} K_{ \pm}^{4},} & {\left[R_{3}, K_{ \pm}^{4}\right]= \pm \frac{1}{2} K_{ \pm}^{4} .}
\end{array}
$$


Comparison of these commutation relations with those of $s o(4,2)$ roots and Cartan generators as given in (A.6) and (A.7) suggests a correspondence between $L_{ \pm}, R_{ \pm}, K_{ \pm}^{i},(i=$ $1,2,3,4)$ and the roots $E_{ \pm\left(e^{i} \pm e^{j}\right)}$ of $s o(4,2)$. This is obvious for the $s o(4)=s u(2)_{L} \oplus s u(2)_{R}$ subalgebra. For this proposed correspondence the operator $A$ needs to be identified with a particular Cartan generator of $s o(4,2)$, as we will lay out in detail in the following section. Nevertheless, the commutation relations among $K_{ \pm}^{i}$, as inferred from those of $M_{a}$ and $N_{a}$ in (2.21) include nonlinear terms in $H$ and $A$ and does not immediately fit into the $s o(4,2)$ commutation relations. For instance, we find

$$
\begin{aligned}
{\left[K_{-}^{1}, K_{+}^{1}\right] } & =4\left(J_{12}+A\right)\left(H+\frac{3}{2} A-1\right)+J_{34}-\sum_{c<d} J_{c d}^{2}+\left(J_{12}+J_{34}\right)\left(J_{12}-J_{34}\right), \\
& =4\left(L_{3}+R_{3}+A\right)\left(H+\frac{3}{2} A-1\right)+\left(L_{3}-R_{3}\right)-A^{2}+\frac{3}{4}+4 L_{3} R_{3} .
\end{aligned}
$$

Such complications are encountered in several different contexts, for instance in the Kepler problem in identifying $\mathrm{SO}(4)$ as the extended dynamical symmetry group of the Hydrogen atom [43]. It was also faced in the $3 D$ case treated in [1]. This issue can be remedied by appropriately scaling the operators $K_{ \pm}^{i}$ acting on the energy eigenstates $\left|n, l^{\prime}, m_{L}, m_{R}\right\rangle$. It turns out that the suitable scalings of $K_{ \pm}^{i}$ can be obtained by exploiting the operator

$$
S=4\left(H+A-\frac{3}{2}\right),
$$

as we will discuss in detail in the next section.

Then, $A, L_{i}, R_{i}$, and

$$
\frac{1}{\sqrt{S}} K_{+}^{i}, \quad K_{-}^{i} \frac{1}{\sqrt{S}}
$$

generate the non-compact group $\mathrm{SO}(4,2)$ on the energy eigenstates $\left|n, l^{\prime}, m_{L}, m_{R}\right\rangle$. Thus, we identify $\mathrm{SO}(4,2)$ as the extended dynamical symmetry of the model described by the Hamiltonian in (2.2). In the next section, by providing the details of this result, we show how the infinite-fold degeneracy of the positive helicity branch can be labeled in terms of a particular discrete UIR of $\mathrm{SO}(4,2)$. We will also see how the finite degeneracy of the negative helicity branch is explained using the same machinery and a related UIR.

\section{Discrete UIRs of $\mathrm{SO}(4,2)$ and the degenerate LL spectrum}

We are interested in the discrete infinite-dimensional UIRs of the $s o(4,2)$ Lie algebra and the corresponding Lie group $\mathrm{SO}(4,2)$. These are usually called the doubletons $[38,39]$ in the literature and they are bounded from below and they can be built via their lowest weight states. Here we provide the essential features and properties of these representations, which are sufficient for our purposes, while some of the details of their construction as well as some formulas for quick reference are relegated to the appendix A and B to make the paper self-contained.

Hermitian generators $M_{\mu \nu}$ of the $s o(4,2)$ Lie algebra satisfy the commutation relations

$$
\left[M_{\mu \nu}, M_{\rho \sigma}\right]=i\left(\eta_{\mu \rho} M_{\nu \sigma}+\eta_{\nu \sigma} M_{\mu \rho}-\eta_{\mu \sigma} M_{\nu \rho}-\eta_{\nu \rho} M_{\mu \sigma}\right)
$$


where the metric is given as $\eta_{\mu \nu}=\operatorname{diag}(1,1,1,1,-1,-1),(\mu, \nu=1, \cdots, 6)$. Cartan subalgebra of $s o(4,2)$ is generated by $\left(H_{1}, H_{2}, H_{3}\right) \equiv\left(M_{12}, M_{34}, M_{56}\right)$. Standard Cartan-Weyl basis can be used to express the generators of $s o(4,2)$. This and the corresponding formulas that relate them to the generators $M_{\mu \nu}$ are provided in appendix A. The subalgebra so(4) of $s o(4,2)$ has the Cartan generators $H_{1}, H_{2}$, while for our purposes it is more convenient to use them in the $s u(2) \oplus s u(2)$ basis, as $L_{3}=\frac{1}{2}\left(H_{1}+H_{2}\right)$ and $R_{3}=\frac{1}{2}\left(H_{1}-H_{2}\right)$. The relevant commutation relations among these generators are also given in appendix A.

In order to present these discrete UIRs $\mathrm{SO}(4,2)$, we exploit the maximally compact subgroup $\mathrm{SU}(2)_{L} \otimes \mathrm{SU}(2)_{R} \otimes \mathrm{U}(1)$, which has the same Cartan subalgebra as that of $\mathrm{SO}(4,2)$. The $\mathrm{U}(1)$ part of this subgroup is generated by $H_{3}=M_{56}$, and it is usually called the conformal Hamiltonian in the literature [38, 39]. With respect to $H_{3}$, the Lie algebra $s o(4,2)$ admits the three-graded decomposition [38, 39]

$$
\text { so }(4,2) \equiv \mathcal{L}^{+} \oplus \mathcal{L}^{0} \oplus \mathcal{L}^{-},
$$

where $\mathcal{L}^{0}$ stands for the maximally compact subalgebra $s u(2)_{L} \oplus s u(2)_{R} \oplus u(1)$ and $\mathcal{L}^{ \pm}$ contain the remaining generators, with the three-grading defined as

$$
\left[\mathcal{L}^{0}, \mathcal{L}^{ \pm}\right]=\mathcal{L}^{ \pm}, \quad\left[H_{3}, \mathcal{L}^{ \pm}\right]= \pm \mathcal{L}^{ \pm}
$$

We may introduce four pairs of annihilation and creation operators, which are split into two "colors", namely $a$ 's and $b$ 's as

$$
\left[a_{\alpha}, a_{\beta}^{\dagger}\right]=\delta_{\alpha \beta}, \quad\left[b_{\alpha}, b_{\beta}^{\dagger}\right]=\delta_{\alpha \beta}, \quad \alpha, \beta=1,2,
$$

acting on the Fock space $\mathcal{F}$ spanned by $\left|n_{a}, n_{b}\right\rangle$. Generators of $\mathrm{SO}(4,2)$ can be expressed in terms of the bilinears of these annihilation-creation operators as given in (B.1) and (B.2) and the three-grading structure is manifest in this basis.

A Schwinger-type realization (as given in appendix B (B.3)-(B.4)) of the so(4,2) using the oscillator algebra and the fundamental spinor IRR of $s o(4,2)$ yields the discrete UIRs of $s o(4,2)$. In particular, Cartan generator $H_{3}$ and a $\mathrm{SO}(4,2)$ invariant operator $\widehat{N}$ take the forms [38-40]:

$$
\begin{aligned}
H_{3} & =M_{56}=\bar{\psi} \Sigma_{56} \psi=\frac{1}{2}\left(\hat{N}_{a}+\hat{N}_{b}+2\right), \\
\hat{N} & :=\bar{\psi} \psi=-\hat{N}_{a}+\hat{N}_{b}-2,
\end{aligned}
$$

where $\hat{N}_{a}=a_{i}^{\dagger} a_{i}$ and $\hat{N}_{b}=b_{i}^{\dagger} b_{i}$ stand for the number operators in the colors $a$ and $b$. It is convenient to label the states in the Fock space $\mathcal{F}$ with the UIRs of the maximally compact subalgebra $s u(2)_{L} \oplus s u(2)_{R} \oplus u(1)$, in the form $\left|h_{3}, J_{L}, J_{R}\right\rangle$, on which any combination of annihilation-creation pairs (given in (B.1), (B.2)) naturally acts. In this notation, the vacuum state is $|1,0,0\rangle$ and annihilated by all $a_{i}$ 's and $b_{i}$ 's. Therefore, it is specified by $N=-2$ eigenvalue of $\widehat{N}$ and has unit conformal energy, i.e. $h_{3}=1$. Based on the vacuum $|1,0,0\rangle$, entire Fock space splits into a direct sum of infinite number of unitary irreducible representations [38, 39], which are labeled by the eigenvalues of $H_{3}$ and $\hat{N}$. In fact, in 
this way, we can write two inequivalent infinite-dimensional families of representations of so $(4,2)$ with the lowest weight vectors, which are given as

$$
\begin{array}{ll}
\left|1+\frac{k}{2}, \frac{k}{2}, 0\right\rangle, \quad N=-k-2, \quad k \in \mathbb{Z}, \\
\left.1+\frac{k}{2}, 0, \frac{k}{2}\right\rangle, \quad N=k-2, \quad k \in \mathbb{Z} .
\end{array}
$$

The fact that these are the lowest weight vectors of a representation of $s o(4,2)$ is easily observed since all $E_{-\left(e^{i} \pm e^{j}\right)} \subset \mathcal{L}^{-}$annihilate these states as they are built up from combinations of $a_{i} b_{j}$. With the action of the ladder operators $E_{ \pm\left(e^{i} \pm e^{j}\right)} \subset \mathcal{L}^{ \pm}$on either of the lowest weights given in (3.6), infinite number of states are generated for any given value of $k$. In other words, for each value of $k$, two inequivalent unitary irreducible representations which are infinite-dimensional are generated in this manner. These are called the doubletons of $s o(4,2)$ and the corresponding group $\mathrm{SO}(4,2)$. Interchanging $s u(2)_{L}$ and $s u(2)_{R}$ swaps these inequivalent doubletons at a given value of $k$. We can label the doubleton representations via the eigenvalue $N=\mp k-2$ of $\widehat{N}$. In order to label all the states in a given doubleton, in addition to the labels $h_{3}, J_{L}, J_{R}$, we also need the eigenvalues of $L_{3}$ and $R_{3}$, which we denote as $m_{L}$ and $m_{R}$. Thus, we label the states as $\left|h_{3}, J_{L}, J_{R}, m_{L}, m_{R}\right\rangle$. Roots in $\mathcal{L}^{ \pm}$shift $h_{3}$ to $h_{3} \pm 1$ and each of $J_{L}, J_{R}$ by $\pm \frac{1}{2}$. This means that the eigenvalue of $\widehat{N}$ is preserved under the action of the roots, that is, $\widehat{N}$ commutes with all the generators, verifying that it is an invariant operator as previously claimed. For the eigenvalues of the operators $H_{3}$ and $\widehat{N}$ in (3.5), we may write in terms of $J_{L}$ an $J_{R}$

$$
\begin{aligned}
h_{3} & =1+J_{L}+J_{R}, \\
N & =-N_{a}+N_{b}-2=-2 J_{L}+2 J_{R}-2=\mp k-2,
\end{aligned}
$$

where $\mp$ sign in the r.h.s. of the last equality on the second line specifies the two inequivalent doubleton representation given in (3.6). Inverting these equations we have,

$$
J_{L}=\frac{1}{2} h_{3} \pm \frac{1}{4} k-\frac{1}{2}, \quad J_{R}=\frac{1}{2} h_{3} \mp \frac{1}{4} k-\frac{1}{2} .
$$

Explicit form of the action of the roots on the states $\left|h_{3}, J_{L}, J_{R}, m_{L}, m_{R}\right\rangle$ is provided in appendix B.

We are now in a position to state one of the most crucial result in this article. Namely, we observe that the infinitely degenerate states of the $4 D$ model at each energy level on either of the chiral components can be labeled by one or the other of the doubleton representations of $\mathrm{SO}(4,2)$ with $N=-3$ or $N=-1$, since for either of the two we can then match the eigenvalues $h_{3}=\frac{3}{2}, \frac{5}{2}, \cdots$ of $H_{3}$ with the eigenvalues $l^{\prime}=\frac{3}{2}, \frac{5}{2}, \cdots$ of the operator $A$. In other words, spectrum of $A$ matches in a one to one and onto manner with that of $H_{3}$ on the states spanning these doubleton representations. Hence, from now on we make the identification $\left|h_{3}, m_{L}, m_{R}\right\rangle \equiv\left|\ell^{\prime}, m_{L}, m_{R}\right\rangle$ for the UIRs with $N=-3$ or $N=-1$. 
Comparing (2.23) with (A.6) and (A.7) we further infer the identifications

$$
\begin{aligned}
& \frac{1}{\sqrt{S}} K_{+}^{1} \equiv E_{e^{1}+e^{3}}, \quad \frac{1}{\sqrt{S}} K_{+}^{2} \equiv E_{-\left(e^{1}-e^{3}\right)}, \quad \frac{1}{\sqrt{S}} K_{+}^{3} \equiv E_{e^{2}+e^{3}}, \quad \frac{1}{\sqrt{S}} K_{+}^{4} \equiv E_{-\left(e^{2}-e^{3}\right)}, \\
& K_{-}^{1} \frac{1}{\sqrt{S}} \equiv E_{-\left(e^{1}+e^{3}\right)}, \quad K_{-}^{2} \frac{1}{\sqrt{S}} \equiv E_{\left(e^{1}-e^{3}\right)}, \quad K_{-}^{3} \frac{1}{\sqrt{S}} \equiv E_{-\left(e^{2}+e^{3}\right)}, \quad K_{-}^{4} \frac{1}{\sqrt{S}} \equiv E_{\left(e^{2}-e^{3}\right)} .
\end{aligned}
$$

Acting on the states $\left|n, l^{\prime}, m_{L}, m_{R}\right\rangle, K_{ \pm}^{i}$ pick an additional factor of $2 \sqrt{n+l^{\prime} \pm \frac{1}{2}}$ for $\ell^{\prime}>0$ as can be seen using (2.24), (3.9) and (B.5). Concretely, we have

$$
\begin{aligned}
L_{ \pm}\left|n, l^{\prime}, m_{L}, m_{R}\right\rangle= & \frac{1}{2} \sqrt{\left(\ell^{\prime} \pm 2 m_{L}+\frac{3}{2}\right)\left(l^{\prime} \mp 2 m_{L}-\frac{1}{2}\right)}\left|n, l^{\prime}, m_{L} \pm 1, m_{R}\right\rangle, \\
R_{ \pm}\left|n, l^{\prime}, m_{L}, m_{R}\right\rangle= & \frac{1}{2} \sqrt{\left(\ell^{\prime} \pm 2 m_{R}+\frac{1}{2}\right)\left(l^{\prime} \mp 2 m_{R}-\frac{3}{2}\right)}\left|n, l^{\prime}, m_{L}, m_{R} \pm 1\right\rangle, \\
K_{ \pm}^{1}\left|n, l^{\prime}, m_{L}, m_{R}\right\rangle= & \sqrt{\left(l^{\prime}+\frac{1}{2}+2 m_{L} \pm 1\right)\left(l^{\prime}-\frac{1}{2}+2 m_{R} \pm 1\right)\left(n+l^{\prime} \pm \frac{1}{2}\right)} \\
& \times\left|n, l^{\prime} \pm 1, m_{L} \pm \frac{1}{2}, m_{R} \pm \frac{1}{2}\right\rangle, \\
K_{ \pm}^{2}\left|n, l^{\prime}, m_{L}, m_{R}\right\rangle= & \sqrt{\left(l^{\prime}+\frac{1}{2}-2 m_{L} \pm 1\right)\left(l^{\prime}-\frac{1}{2}-2 m_{R} \pm 1\right)\left(n+l^{\prime} \pm \frac{1}{2}\right)} \\
& \times\left|n, l^{\prime} \pm 1, m_{L} \mp \frac{1}{2}, m_{R} \mp \frac{1}{2}\right\rangle, \\
K_{ \pm}^{3}\left|n, l^{\prime}, m_{L}, m_{R}\right\rangle= & \sqrt{\left(l^{\prime}+\frac{1}{2}+2 m_{L} \pm 1\right)\left(l^{\prime}-\frac{1}{2}-2 m_{R} \pm 1\right)\left(n+l^{\prime} \pm \frac{1}{2}\right)} \\
& \times\left|n, l^{\prime} \pm 1, m_{L} \pm \frac{1}{2}, m_{R} \mp \frac{1}{2}\right\rangle, \\
K_{ \pm}^{4}\left|n, l^{\prime}, m_{L}, m_{R}\right\rangle= & \sqrt{\left(l^{\prime}+\frac{1}{2}-2 m_{L} \pm 1\right)\left(l^{\prime}-\frac{1}{2}+2 m_{R} \pm 1\right)\left(n+l^{\prime} \pm \frac{1}{2}\right)} \\
& \times\left|n, l^{\prime} \pm 1, m_{L} \mp \frac{1}{2}, m_{R} \pm \frac{1}{2}\right\rangle .
\end{aligned}
$$

The foregoing discussion makes the identification of the extended symmetry generators with either of the $\mathrm{SO}(4,2)$ doubleton representation with $N=-3$ or $N=-1$ manifest and either of the representations can be used to enumerate the infinite fold degeneracy of the flat LL spectra of the model given in (2.16).

For $\ell^{\prime}<0$, i.e. the negative helicity component of the spectrum, energy levels are only finitely degenerate. We easily see from (2.16) that at $E=3$, only possible value of $l^{\prime}$ is $-\frac{3}{2}$, while for $E=4$, the possible values for $\ell^{\prime}$ are $-\frac{3}{2}$ and $-\frac{5}{2}$ and in general for $E \geq 3$ the possible values of $\ell^{\prime}$ are $-\frac{3}{2},-\frac{5}{2}, \cdots,\left(\frac{3}{2}-E\right)$. To label these degenerate states, we essentially need the representations defined through their highest weight states, i.e. the complex conjugate representation. The latter can be obtained from the doubletons defined 
via (3.6) by taking $\left(h_{3}, k\right) \rightarrow\left(-h_{3},-k\right)$ and making the exchange ${ }^{1} J_{L} \leftrightarrow J_{R}$. Complex conjugate doubleton representations are bounded from above and those with $N=-3^{*}$ and $N=-1^{*}$ are the two inequivalent UIRs that may be used. The physical operators $K_{ \pm}^{i}$ acting on these complex conjugate representations bring a factor $\sqrt{E+l^{\prime}-1 \pm \frac{1}{2}}$, where $E$ stands for the energy eigenvalue. ${ }^{2}$ We see that the $K_{-}^{i}$ annihilate the states with $l^{\prime} \leq \frac{3}{2}-E$, with $E=3,4, \cdots$, fitting perfectly with the observed finitely degenerate spectrum.

Let us also recall that the spin operator $S_{a b}$ have the chiral components $S_{a b}^{+}$and $S_{a b}^{-}$ and the spectrum (2.16) is the same in each chiral branch. These chiral parts are mapped to each other upon interchanging the left- and the right- generators of $\mathrm{SU}(2)_{L} \times \mathrm{SU}(2)_{R}$. From these facts, we immediately infer that, we can employ both of the doubleton representations with $N=-3$ and $N=-1$ one enumerating the infinite degeneracy in the left-chiral and the other in the right-chiral component for the flat spectra with positive helicity. Similarly both of the complex conjugate UIRs with $N=-3^{*}$ and $N=-1^{*}$ can be employed to label the negative helicity part of the spectrum. In this manner all the degeneracies in the spectrum (2.16) are accounted for.

\section{Generalization to $d$-dimensions}

It is essentially rather straightforward to generalize the $4 D$ model and the preceding developments to $d$-dimensions. With the $\mathrm{SO}(d)$ gauge field $G=2 m \omega r_{b} S_{a b},(2.1)$ generalizes to

$$
H_{d}=\frac{1}{2 m}\left(p_{a}-G_{a}\right)^{2}-\frac{d-2}{2} m \omega^{2} r_{a}^{2}
$$

while (2.2) has the same formal structure

$$
H_{d}=\frac{p_{a}^{2}}{2 m}+\frac{1}{2} m \omega^{2} r_{a}^{2}-\omega \sum_{a<b=1}^{d} L_{a b} \Gamma_{a b}
$$

where now $L_{a b}:=r_{a} p_{b}-r_{b} p_{a},(a, b=1, \cdots, d)$ are the orbital angular momentum operators that span the $(l, 0, \cdots, 0)$ IRR of $\mathrm{SO}(d)$, which is of dimension $\mathcal{N}=(d+2 l-2) \frac{(d+l+3) !}{l !(d-2) !}$. The Casimir operator in this IRR of $\mathrm{SO}(d)$ satisfies $\sum_{a<b} L_{a b}^{2}=l(l+d-2) \mathbb{1}_{\mathcal{N}} . \Gamma_{a b}$ are proportional to the spin operator $S_{a b}$ in $d$-dimensions and can be given in terms of the commutators of the $\Gamma$-matrices in $d$-dimensions as $S_{a b}:=\frac{1}{2} \Gamma_{a b}:=-\frac{i}{4}\left[\Gamma_{a}, \Gamma_{b}\right]$. For $d$ odd, $d=2 k+1, \Gamma_{a}$ are of rank $k$; they are $2^{k} \times 2^{k}$ matrices and there are $2 k+1$ of them. $\mathrm{SO}(2 k+1)$ has rank $k$ and $S_{a b}$ spans the fundamental spinor representation $\left(\frac{1}{2}, \frac{1}{2}, \cdots, \frac{1}{2}\right)$ of $\mathrm{SO}(2 k+1)$ which is $2^{k} \times 2^{k}$-dimensional. For $d$ even, $d=2 k+2, \Gamma_{a}$ has rank $k, 2^{k+1} \times 2^{k+1}$ matrices and $S_{a b}$ span a reducible representation of $\mathrm{SO}(2 k+2)$, which decomposes as $S_{a b}=S_{a b}^{+} \oplus S_{a b}^{-}$

\footnotetext{
${ }^{1}$ To be more precise, these representations are generated by $-M_{a b}^{*}$.

${ }^{2}$ Note that, in terms of the energy eigenvalues, the factor $\sqrt{\left(n+l^{\prime} \pm \frac{1}{2}\right)}$ that appears in (3.10) also takes the form $\sqrt{E+l^{\prime}-1 \pm \frac{1}{2}}$. We already know that, $K_{ \pm}^{i}$ are commuting with the Hamiltonian, however the negative helicity part of the spectrum is not independent of $l^{\prime}$, therefore it is imperative to express this factor in terms of the energy to make the proper physical interpretation manifest, while it makes no difference to write it in terms of $n$ or $E$ for the positive helicity part as $E$ does not depend on $l^{\prime}$.
} 
to the fundamental left- and right-chiral spinor representations $\left(\frac{1}{2}, \frac{1}{2}, \cdots, \pm \frac{1}{2}\right)$, which are each $2^{k} \times 2^{k}$-dimensional. The chiral projections to $S_{a b}^{ \pm}$can be obtained using the projection operators $\mathcal{P}^{ \pm}=\frac{1}{2}\left(1 \pm \Gamma_{2 k+3}\right)$, where $\Gamma_{2 k+3}:=\Gamma_{1} \Gamma_{2} \cdots \Gamma_{2 k+2}$. In terms of the $\Gamma$-matrices of rank $k$, we may write $S_{a b}^{ \pm} \equiv\left(S_{i j}, S_{i 2 k+2}\right):=\left(S_{i j}, \pm \frac{1}{2} \Gamma_{i}\right)$ with $i, j=1, \cdots, 2 k+1$. The facts listed above are well-known and the spectrum of $H$ is already given in [15]. For completeness, we provide the essential results here, to lay out the foundations for the developments that will ensue. Eigenvalues of the SO term follows from a similar calculation as in the $4 D$ case and they are given as

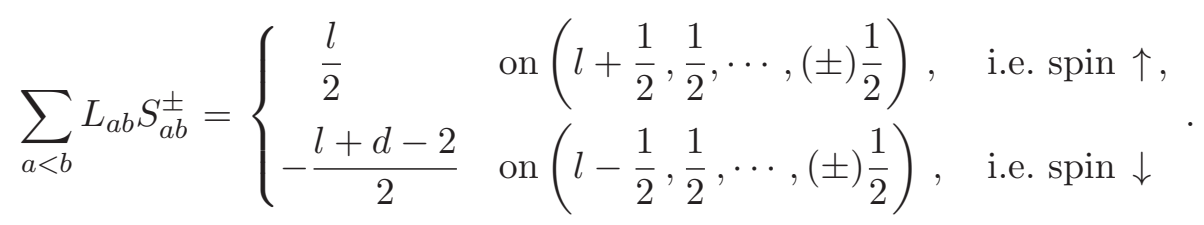

It should be clear that the $( \pm)$ in (4.3) distinguishes the left- and the right-chiral representations for $\mathrm{SO}(2 k+2)$; while for $\mathrm{SO}(2 k+1)$ only the upper sign appears. This gives the spectrum of $H_{d}$ in (4.1) or equally in (4.2) as

$$
E=\left\{\begin{array}{ll}
2 \omega\left(n+\frac{d}{4}\right), & \operatorname{spin} \uparrow \\
2 \omega\left(n+l+\frac{3}{4} d-1\right)=2 \omega\left(n-l^{\prime}+\frac{d}{4}+\frac{1}{2}\right), & \text { spin } \downarrow
\end{array} .\right.
$$

Note that as in the $4 D$ model, the spectrum in the positive helicity branch is flat, indicating an infinite-fold degeneracy for this part of the spectrum. We also stick to the choice of the parameter values $m=1$ and $\omega=\frac{1}{2}$. In analogy with the $3 D[1]$ and $4 D$ results, we may introduce the operator

$$
A=\sum_{a<b} L_{a b} \Gamma_{a b}+\frac{d-1}{2},
$$

whose eigenvalues may still be denoted as $l^{\prime}$. We have $l^{\prime}=l+\frac{d-1}{2}$ for the positive and $l^{\prime}=-l-\frac{d-3}{2}$ for the negative helicity components so that $l^{\prime}= \pm \frac{d-1}{2}, \pm \frac{d+1}{2}, \cdots$. The eigenstates of the Hamiltonian $H_{d}$ can be denoted by the kets $\left|n, l^{\prime},[s]_{\mathrm{SO}(d)},[m]_{\mathrm{SO}(d)}\right\rangle$, where $[s]_{\mathrm{SO}(d)}$ stands as a collective index for the $\mathrm{SO}(d)$ UIR and $[m]_{\mathrm{SO}(d)}$ as a collective index of the quantum numbers within this UIR of $\mathrm{SO}(d)$ that unambiguously label these eigenstates.

Using the operator $A$, we can introduce the $d$-dimensional vector operators $M_{a}$ and $N_{a}$ exactly in the same formal form as given in (2.17), except that the indices $a, b$ are now taking values in the interval $(1, \cdots, d)$. Total angular momentum operators $J_{a b}=L_{a b}+S_{a b}$, $A$ and appropriately scaled linear combinations of $M_{a}, N_{a}$ span $\frac{1}{2}(d+2)(d+1)$-dimensional group, which can be identified with the non-compact group $\mathrm{SO}(d, 2)$. This essentially works in the same manner, as we have laid out in detail for the $4 D$ case. We may use the discrete unitary irreducible representations of $\mathrm{SO}(d, 2)$ defined through their lowest weight vectors to label the infinite degeneracy of the flat part of the spectrum in (4.4). so(d,2) is of rank $k+1$ for $d=2 k+1$ and of rank $k+2$ for $d=2 k+2$. The relevant discrete UIR of $s o(d, 2)$ 
can be constructed using the three-graded decomposition of the Lie algebra so(d,2) w.r.t. its maximally compact subalgebra [41, 42]

$$
s o(d, 2) \equiv \mathcal{L}^{+} \oplus \mathcal{L}^{0} \oplus \mathcal{L}^{-},
$$

where $\mathcal{L}^{0}$ stands for the maximally compact subalgebra $s o(d) \oplus u(1) . \mathcal{L}^{ \pm}$which contains the remaining generators of $s o(d, 2)$, and the three-grading has the same structure as defined before in (3.3). From the existing literature, it is readily known that the discrete unitary representations of $s o(d, 2)$ can be labeled by the eigenvalues of the U(1)-generator and these representations generalize the singleton representation of $s o(3,2)$ for odd values of $d$ and the doubleton representations of $s o(4,2)$ for even values of $d[41,42]$. For $d=2 k+2$, the representations we need fall into the class in which the $\mathrm{SO}(2 k+2)$ subgroup carry the IRRs $\left(\frac{s}{2}, \frac{s}{2}, \cdots \pm \frac{s}{2}\right) \equiv[s]_{\mathrm{SO}(d)}$, where $s$ is a non-negative integer. Corresponding to each of these representations there is a UIR of $s o(d, 2)$ with the lowest weight vectors $\left|\frac{1}{2}(d+s-2),\left(\frac{s}{2}, \frac{s}{2}, \cdots \pm \frac{s}{2}\right)\right\rangle$, whose $\mathrm{U}(1)$-charge, i.e. the eigenvalue of the $(k+2)^{t h}$ Cartan generator $H_{k+2}$ is $\frac{1}{2}(d+s-2)$. Action of the operators in the $\mathcal{L}^{-}$sector of the three-grading annihilate these lowest weight states, while the repeated action of operators $\mathcal{L}^{+}$generates these UIRs. In particular, action of $\mathcal{L}^{ \pm}$shifts the eigenvalue of $H_{k+2}$ by \pm 1 , and map $[s]_{\mathrm{SO}(d)}$ to $[s \pm 1]_{\mathrm{SO}(d)}$, while also changing the collective $[m]_{\mathrm{SO}(d)}$ indices; $\mathcal{L}^{ \pm}$ can be spanned by the roots of $s o(d, 2)$ in the Cartan-Weyl basis, whose organization is determined by the fact that roots in $\mathcal{L}^{ \pm}$should shift the eigenvalue of $H_{k+2}$ by \pm 1 .

Among these UIRs, we need the one with $s=1$, whose U(1) charge in the lowest weight sector matches with the lowest possible positive eigenvalue $\frac{d-1}{2}$ of $A$. Thus, the spectrum of $A$ matches with that of $H_{k+2}$ on these UIRs and the states in either of them with the lowest weights $\left|\frac{1}{2}(d-1),\left(\frac{1}{2}, \frac{1}{2}, \cdots \pm \frac{1}{2}\right)\right\rangle$ span the infinite degeneracy of the flat spectrum in (4.4). There are overall $2 d$ different linear combinations $K_{ \pm}^{i}(i: 1, \cdots, d)$ of $M_{a}$ 's and $N_{a}$ 's such that $\frac{1}{\sqrt{S}} K_{+}^{i} \operatorname{span} \mathcal{L}^{+}$and $K_{-}^{i} \frac{1}{\sqrt{S}}$ span $\mathcal{L}^{-}$, where $S=H+A-\frac{1}{2}\left(\frac{d}{2}+1\right)$ up to an overall constant which is immaterial for our present purposes. Just like the $4 D$ case, we can associate one of these UIRs with the left- and the other with the right- chiral component to label and distinguish the degenerate spectrum. For the negative helicity states, energy disperses with the eigenvalues of $A$ and the degeneracy is finite. The complex conjugate representations, which are practically obtained by $h_{k+2} \rightarrow-h_{k+2}$, have the highest weight vectors $\left|-\frac{1}{2}(d-1),\left(\frac{1}{2}, \frac{1}{2}, \cdots \pm \frac{1}{2}\right)\right\rangle$ and can be used to label the degenerate states in this branch, noting that the unscaled operators $K_{-}^{i}$ annihilate the states with $l^{\prime}<\frac{d}{4}+\frac{1}{2}-E$, with $E$ taking on the values $\frac{3}{4} d, \frac{3}{4} d+1, \cdots$. For $d=4$, our previously determined result is immediately obtained, while, for instance, for $d=6$, negative helicity states have the lowest energy $\frac{9}{2}$ and therfore no states with $l^{\prime}<-\frac{5}{2}$ exist, in perfect agreement with the observed spectrum and degeneracy of the negative helicity states.

For odd values of $d$, the relevant representation of $s o(d, 2)$ is also labeled by the $\mathrm{U}(1)$ charge, and has the value $\frac{d-1}{2}$ for the lowest weight state [41, 42]. The latter is given by $\left|\frac{1}{2}(d-1),\left(\frac{1}{2}, \frac{1}{2}, \cdots \frac{1}{2}\right)\right\rangle$, where the $\left(\frac{1}{2}, \frac{1}{2}, \cdots \frac{1}{2}\right)$ is the $2^{\left(\frac{d-1}{2}\right)}$-dimensional fundamental spinor IRR of $\mathrm{SO}(d)$. These states are annihilated by all the operators belonging to $\mathcal{L}^{-}$ of the three grading and a UIR of $s o(d, 2)$ is generated by the repeated application of 
the operators in $\mathcal{L}^{+}$. It can be readily noted that, as opposed to the infinite family of representations for even $d$ (corresponding to the pair of UIRs labeled by the integer $s$ ), for $d$ odd there is only a unique spinoral UIR of $s o(d, 2)$. For $d=3$, this is nothing but the Dirac singleton representation with spin $\frac{1}{2}$. In this UIR spectrum of $A$ identifies with that of the $\mathrm{U}(1)$ generator, which may be taken as the $(k+1)^{t h}$ Cartan generator $H_{k+1}$, and the states generated from the lowest weight $\left|\frac{1}{2}(d-1),\left(\frac{1}{2}, \frac{1}{2}, \cdots \frac{1}{2}\right)\right\rangle$ completely label the infinite degeneracy of the flat spectrum. The rest of the correspondence is almost the same as that of even $d$ given in the previous paragraph, except that for $d$ odd, the operator $K_{ \pm}^{2 k+1}$ associated to the last root pair $E_{ \pm e^{k}}$, in the form $E_{e^{k}} \equiv \frac{1}{\sqrt{S}} K_{+}^{2 k+1}$ and $E_{-e^{k}} \equiv K_{i}^{2 k+1} \frac{1}{\sqrt{S}}$ are obtained from the linear combinations of only the $(2 k+1)^{t h}$ components of $M_{a}$ and $N_{a}$, i.e. $K_{ \pm}^{2 k+1}=\frac{1}{\sqrt{2}}\left(M_{2 k+1} \mp i N_{2 k+1}\right)$, while the other $K_{ \pm}^{i}$ are linear combinations that involve two components from each of $M_{a}$ and $N_{a}$ (2.23). Thus, all $K_{ \pm}^{i}$ shift the eigenvalue of $H_{k+1}$ by \pm 1 , and $[s]_{\mathrm{SO}(d)}$ to $[s \pm 1]_{\mathrm{SO}(d)}$, while $[m]_{\mathrm{SO}(d)}$ change accordingly under $K_{ \pm}^{-}$, $(i \neq 2 k+1)$, but remains unchanged under $K_{ \pm}^{2 k+1}$. For $d=3$, this result can be seen from the formula provided in [1] and is a characteristic discriminating odd $d$ from even $d$.

\section{Spectrum generating algebra}

Using the annihilation and creation operators

$$
c_{a}=\frac{1}{2} r_{a}-i p_{a}, \quad c_{a}^{\dagger}=\frac{1}{2} r_{a}+i p_{a}, \quad\left[c_{a}, c_{b}^{\dagger}\right]=\delta_{a b}, \quad(a=1, \cdots, d),
$$

we may express the Hamitonian $H_{d}$ in (4.2) as

$$
H=\frac{1}{2}\left(\widehat{N}_{c}+\frac{d}{2}\right)-\frac{1}{2} \sum_{a<b} L_{a b} \Gamma_{a b},
$$

where $\widehat{N}=c_{a}^{\dagger} c_{a}$ and the orbital angular momentum operator can be expressed in terms of $c_{a}, c_{a}^{\dagger}$ as $L_{a b}=-i\left(c_{a}^{\dagger} c_{b}-c_{b}^{\dagger} c_{a}\right)$.

Generalizing the discussion given in [1], we may introduce the operators $\mathcal{S}_{ \pm}$, which are quadratic in $c_{a}$ 's and $c_{a}^{\dagger}$ 's as

$$
\mathcal{S}_{+}=-\frac{1}{2} c_{a}^{\dagger} c_{a}^{\dagger}, \quad \mathcal{S}_{-}=-\frac{1}{2} c_{a} c_{a} .
$$

Since, $\left[\hat{N}, \mathcal{S}_{ \pm}\right]= \pm 2 \mathcal{S}_{ \pm}$and $\left[L_{a b}, \mathcal{S}_{ \pm}\right]=0$, we infer that $\mathcal{S}_{ \pm}$shifts the energy eigenvalues by \pm 1 . It is straightforward to show that

$$
\left[H, \mathcal{S}_{ \pm}\right]= \pm \mathcal{S}_{ \pm}, \quad\left[\mathcal{S}_{+}, \mathcal{S}_{-}\right]=-2\left(H+\frac{A}{2}-\frac{d-1}{4}\right)
$$

Upon identification of $H+\frac{A}{2}-\frac{d-1}{4}$ with the $\mathcal{S}_{3}$ generator, (5.4) corresponds to the $\operatorname{SO}(2,1)$ commutation relations. In particular, $\mathcal{S}_{3}, \mathcal{S}_{ \pm}$span the unitary irreducible representation of $\mathrm{SO}(2,1)$ with the extremal weights $\Lambda=\frac{l^{\prime}}{2}+\frac{1}{4}$ for $\ell^{\prime}=\frac{d-1}{2}, \frac{d+1}{2}, \cdots$ and $\Lambda=-\frac{l^{\prime}}{2}+\frac{3}{4}$ for $l^{\prime}=-\frac{d-1}{2},-\frac{d+1}{2}, \cdots$. In these representations of $\mathrm{SO}(2,1)$, eigenvalues of the Casimir 
operator $\frac{1}{2}\left(\mathcal{S}_{+} \mathcal{S}_{-}+\mathcal{S}_{-} \mathcal{S}_{+}\right)-\mathcal{S}_{3}^{2}$ are given as $\Lambda(1-\Lambda)$. Using this information and (5.4) we easily find

$$
\begin{aligned}
\mathcal{S}_{ \pm}\left|n, l^{\prime}, m_{L}, m_{R}\right\rangle & =\sqrt{\left(n+\frac{1}{2} \pm \frac{1}{2}\right)\left(n+l^{\prime} \pm \frac{1}{2}\right)}\left|n \pm 1, l^{\prime}, m_{L}, m_{R}\right\rangle, \quad l^{\prime}>0 \\
\mathcal{S}_{ \pm}\left|n, l^{\prime}, m_{L}, m_{R}\right\rangle & =\sqrt{\left(n+\frac{1}{2} \pm \frac{1}{2}\right)\left(n-l^{\prime}+1 \pm \frac{1}{2}\right)}\left|n \pm 1, l^{\prime}, m_{L}, m_{R}\right\rangle, l^{\prime}<0 \\
& \equiv \sqrt{\left(E+l^{\prime}-\frac{d}{4} \pm \frac{1}{2}\right)\left(E-\frac{d}{4}+\frac{1}{2} \pm \frac{1}{2}\right)}\left|E \pm 1, l^{\prime}, m_{L}, m_{R}\right\rangle .
\end{aligned}
$$

General considerations on the UIRs of $\mathrm{SO}(2,1)$ require that $\Lambda \geq \frac{1}{2}$ [45], and this is fulfilled in the present case since $\left|l^{\prime}\right| \geq \frac{d-1}{2}$. For both the positive and negative helicity components the lowest weight state is $\left|0, l^{\prime}, m_{L}, m_{R}\right\rangle$. Since the energy spectrum is $E=n-l^{\prime}+\frac{d}{4}+\frac{1}{2}$ for negative helicity states, it is readily inferred from the second line of (5.6) that $\mathcal{S}_{-}$ annihilates the states with $l^{\prime}<\frac{d}{4}+\frac{1}{2}-E$ in accord with the result determined in the preceding section.

\section{Discussion and conclusions}

In this paper we have examined the degeneracies in the energy spectrum of $d \geq 4$ dimensional SHOs coupled to Aharanov-Casher type $\mathrm{SO}(d)$ gauge fields. The Hamiltonians of these models can equally be expressed as SHOs, with a spin-orbit terms, whose coupling strength is tuned to the SHO frequency. With our choice of sign for the SO coupling the positive helicity part of the energy spectrum is flat and led to the interpretation of these models as TR invariant LLs in higher dimensions [15], generalizing the QSHE [3]. Focusing on the $4 D$ model, we have introduced two vector operators commuting the Hamiltonian and succeeded in demonstrating that the symmetry group $\mathrm{SO}(4)$ of the model extends to the non-compact dynamical symmetry $\mathrm{SO}(4,2)$ and that the discrete UIRs of this group, the so called doubletons specified via the invariants $N=-3$ and $N=-1$ provide the complete labeling of the infinite degeneracy of the flat spectrum. Subsequently, all of these results generalized to the models in $d$-dimensions and shown that the extended non-compact symmetry group is indeed $\mathrm{SO}(d, 2)$ and the infinite degeneracy of the flat spectra is completely accounted for by exploiting the discrete series UIRs of $\mathrm{SO}(d, 2)$, which generalize the singleton and the doubleton representations in $d=3$ and $d=4$ to all odd and even dimensions, respectively.

Since $\mathrm{SO}(d, 2)$ is the isometry group of $A d S_{d+1}$, we may contemplate that the LL states are essentially organized w.r.t. an underlying radially "deformed" AdS geometry rather than the flat space, due to the extended non-linear symmetry generated by the operators $J_{a b}, A$ and $K_{ \pm}^{i}$, whose commutation relations involve non-linear terms as manifestly seen from (2.21) and (2.24). The spectrum of the operator $A$ in (4.5) matches with that of the $\mathrm{U}(1)$ generator in the UIR of $\mathrm{SO}(d, 2)$ in which the latter has the eigenvalue $\frac{d-1}{2}$ in the lowest weight states. Considering the $\mathrm{SO}(d, 2)$ as either the conformal group for $d$ dimensional Minkowski space-time or, as above, the isometry group of $A d S_{d+1}$, spectrum 
of the U(1) generator corresponds to the conformal energy or the AdS energy [41, 42]. Under the action of $s o(d, 2)$ ladder operators, eigenvalues of $A$ shift by \pm 1 within the degenerate states, giving further evidence toward the aforementioned interpretation.

Finally, let us note that imposing open boundary conditions say at radius $R_{0}$, the flat spectrum is no longer maintained once the angular momentum value exceeds a critical value, which depends on the LL and can be numerically estimated for a given model, as it was done for $3 D$ case in [15]. Starting around this critical value, the energy spectrum becomes dispersive indicating the emergence of states localized on the boundary [15]. In fact, the energy spectrum at the surface can be linearized around the Fermi angular momentum and becomes essentially governed by the Hamiltonian (see [44] for Dirac operators on spheres)

$$
\begin{aligned}
H_{\text {surface }} & =\frac{v_{F}}{R_{0}} \sum_{a<b} L_{a b} \Gamma_{a b}-\mu \\
& =\frac{v_{F}}{R_{0}}\left(A-\frac{d-1}{2}\right)-\mu,
\end{aligned}
$$

where $\mu$ stands for the chemical potential. Although $\mathrm{SO}(d, 2)$ can no longer be considered as the precise extended dynamical symmetry in the presence of the boundary, (6.1) shifts by $\pm \frac{v_{F}}{R_{0}}$ under the action of $s o(d, 2)$ ladder operators, which suggests the interpretation of $\mathrm{SO}(d, 2)$ as an effective spectrum generating algebra for the surface states. We think that these brief remarks merit further study and any future progress on them will be reported elsewhere.

\section{Acknowledgments}

Part of S.K.'s work was carried out during his sabbatical stay at the physics department of CCNY of CUNY and he thanks V.P. Nair and D. Karabali for the warm hospitality at CCNY and the metropolitan area. S.K. also thanks A.P. Balachandran for discussions and reading the manuscript. S.K. acknowledges the financial support of the Turkish Fulbright Commission under the visiting scholar program and the METU research project GAP-1052018-2809. G. $\ddot{U}$ acknowledges the support of the TUBITAK 2218 post doctoral scholarship program.

\section{A $s o(4,2)$ Lie algebra in the Cartan-Weyl basis}

In this appendix we collect some the standard formulas for $s o(4,2)$ Lie algebra and some details regarding the construction of their doubleton UIRs.

Cartan subalgera of $s o(4,2)$ is spanned by $\left(H_{1}, H_{2}, H_{3}\right) \equiv\left(M_{12}, M_{34}, M_{56}\right)$. Introducing the three-component unit vectors $e^{1}, e^{2}, e^{3}$ with $\left(e^{i}\right)_{j}=\delta_{j}^{i}$, the roots may be expressed as

$$
E_{ \pm\left(e^{1}-e^{2}\right)}, \quad E_{ \pm\left(e^{2}-e^{3}\right)}, \quad E_{ \pm\left(e^{2}+e^{3}\right)}, \quad E_{ \pm\left(e^{1}+e^{2}\right)}, \quad E_{ \pm\left(e^{1}-e^{3}\right)}, \quad E_{ \pm\left(e^{1}+e^{3}\right)} .
$$

It is common to introduce the notation $E_{ \pm \alpha^{\mu}}$ for the roots, with the labels given as $\pm \alpha^{\mu}:=$ $\pm\left(e^{i} \pm e^{j}\right)$ with $i<j$. In a standard short-hand notation of the Cartan-Weyl basis, 
commutation relations among the generators can be compactly expressed as [46]

$$
\begin{aligned}
{\left[H_{i}, H_{j}\right] } & =0, \quad\left[H_{i}, E_{\alpha^{\mu}}\right]=\alpha_{i}^{\mu} E_{\alpha^{\mu}}, \\
{\left[E_{\alpha^{\mu}}, E_{\alpha^{\nu}}\right] } & = \begin{cases}N_{\alpha^{\mu} \alpha^{\nu}} E_{\alpha^{\mu}+\alpha^{\nu}}, & \text { if } \alpha^{\mu}+\alpha^{\nu} \text { is a root } \\
\left(E_{\alpha^{\mu}}, E_{-\alpha^{\mu}}\right) \alpha_{i}^{\mu} H_{i}, & \text { if } \alpha^{\mu}+\alpha^{\nu}=0, \text { and sum over } i \text { is implied, } \\
0, & \text { otherwise }\end{cases}
\end{aligned}
$$

where

$$
\left(E_{\alpha^{\mu}}, E_{\alpha^{\nu}}\right)=\frac{1}{2} \operatorname{Tr} E_{\alpha^{\mu}} E_{\alpha^{\nu}}
$$

Roots listed in (A.1) can be expressed as linear combinations of the $M_{\mu \nu}$ as:

$$
\begin{aligned}
& E_{ \pm\left(e^{1}-e^{2}\right)}=\frac{1}{2}\left(\mp i M_{13}+M_{23}-M_{14} \mp i M_{24}\right) \\
& E_{ \pm\left(e^{2}-e^{3}\right)}=\frac{1}{2}\left(M_{35} \pm i M_{45}-M_{46} \pm i M_{36}\right) \\
& E_{ \pm\left(e^{2}+e^{3}\right)}=\frac{1}{2}\left(M_{35} \pm i M_{45}+M_{46} \mp i M_{36}\right) \\
& E_{ \pm\left(e^{1}+e^{2}\right)}=\frac{1}{2}\left( \pm i M_{23}+M_{13} \pm i M_{14}-M_{24}\right) \\
& E_{ \pm\left(e^{1}-e^{3}\right)}=\frac{1}{2}\left( \pm i M_{25}-M_{26}+M_{15} \pm i M_{16}\right) \\
& E_{ \pm\left(e^{1}+e^{3}\right)}=\frac{1}{2}\left(\mp i M_{25}-M_{26}-M_{15} \pm i M_{16}\right)
\end{aligned}
$$

For the normalized traces we have

$$
\operatorname{Tr} M_{\mu \nu} M_{\rho \sigma}=2 \eta_{\mu \rho} \eta_{\nu \sigma}-2 \eta_{\mu \sigma} \eta_{\nu \rho}
$$

which is consistent with (A.3) as can be easily checked. Using the basis $\left(L_{3}, R_{3}, H_{3}\right)$ with $L_{3}=\frac{1}{2}\left(H_{1}+H_{2}\right)$ and $R_{3}=\frac{1}{2}\left(H_{1}-H_{2}\right)$ for the Cartan subalgebra, the relevant part of the commutation relations among the generator is summarized below for easy reference:

$$
\begin{aligned}
& {\left[L_{3}, E_{ \pm\left(e^{1}-e^{2}\right)}\right]=0,} \\
& {\left[L_{3}, E_{\mp\left(e^{2}-e^{3}\right)}\right]=\mp \frac{1}{2} E_{\mp\left(e^{2}-e^{3}\right)},} \\
& {\left[R_{3}, E_{ \pm\left(e^{1}-e^{2}\right)}\right]= \pm E_{ \pm\left(e^{1}-e^{2}\right)},} \\
& {\left[L_{3}, E_{ \pm\left(e^{2}+e^{3}\right)}\right]= \pm \frac{1}{2} E_{ \pm\left(e^{2}+e^{3}\right)},} \\
& {\left[R_{3}, E_{\mp\left(e^{2}-e^{3}\right)}\right]= \pm \frac{1}{2} E_{\mp\left(e^{2}-e^{3}\right)},} \\
& {\left[L_{3}, E_{ \pm\left(e^{1}+e^{2}\right)}\right]= \pm E_{ \pm\left(e^{1}+e^{2}\right)},} \\
& {\left[R_{3}, E_{ \pm\left(e^{2}+e^{3}\right)}\right]=\mp \frac{1}{2} E_{ \pm\left(e^{2}+e^{3}\right)},} \\
& {\left[L_{3}, E_{\mp\left(e^{1}-e^{3}\right)}\right]=\mp \frac{1}{2} E_{\mp\left(e^{1}-e^{3}\right)},} \\
& {\left[R_{3}, E_{ \pm\left(e^{1}+e^{2}\right)}\right]=0} \\
& {\left[L_{3}, E_{ \pm\left(e^{1}+e^{3}\right)}\right]= \pm \frac{1}{2} E_{ \pm\left(e^{1}+e^{3}\right)},} \\
& {\left[R_{3}, E_{\mp\left(e^{1}-e^{3}\right)}\right]=\mp \frac{1}{2} E_{\mp\left(e^{1}-e^{3}\right)},} \\
& {\left[R_{3}, E_{ \pm\left(e^{1}+e^{3}\right)}\right]= \pm \frac{1}{2} E_{ \pm\left(e^{1}+e^{3}\right)},} \\
& {\left[H_{3}, E_{ \pm\left(e^{1}-e^{2}\right)}\right]=0,} \\
& {\left[E_{\left(e^{1}-e^{2}\right)}, E_{-\left(e^{1}-e^{2}\right)}\right]=H_{1}-H_{2},} \\
& {\left[H_{3}, E_{\mp\left(e^{2}-e^{3}\right)}\right]= \pm E_{\mp\left(e^{2}-e^{3}\right)},} \\
& {\left[E_{\left(e^{2}-e^{3}\right)}, E_{-\left(e^{2}-e^{3}\right)}\right]=-H_{2}+H_{3} \text {, }} \\
& {\left[H_{3}, E_{ \pm\left(e^{2}+e^{3}\right)}\right]= \pm E_{ \pm\left(e^{2}+e^{3}\right)}, \quad\left[E_{\left(e^{2}+e^{3}\right)}, E_{-\left(e^{2}+e^{3}\right)}\right]=-H_{2}-H_{3},} \\
& {\left[H_{3}, E_{ \pm\left(e^{1}+e^{2}\right)}\right]=0 \text {, }} \\
& {\left[E_{\left(e^{1}-e^{3}\right)}, E_{-\left(e^{1}-e^{3}\right)}\right]=-H_{1}+H_{3},} \\
& {\left[H_{3}, E_{\mp\left(e^{1}-e^{3}\right)}\right]= \pm E_{\mp\left(e^{1}-e^{3}\right)}, \quad\left[E_{\left(e^{1}+e^{3}\right)}, E_{-\left(e^{1}+e^{3}\right)}\right]=-H_{1}-H_{3} \text {, }} \\
& {\left[H_{3}, E_{ \pm\left(e^{1}+e^{3}\right)}\right]= \pm E_{ \pm\left(e^{1}+e^{3}\right)}, \quad\left[E_{\left(e^{1}+e^{2}\right)}, E_{-\left(e^{1}+e^{2}\right)}\right]=H_{1}+H_{2} \text {. }}
\end{aligned}
$$




\section{B Details of the construction of doubletons}

With respect to the three-grading of $s o(4,2)$ given in (3.2) and (3.3), out of the six pairs of roots $E_{ \pm\left(e^{i} \pm e^{j}\right)}$, we have $L_{ \pm}=E_{ \pm\left(e^{1}+e^{2}\right)}$ generating $s u(2)_{L}$ together with $L_{3}$ and $E_{ \pm\left(e^{1}-e^{2}\right)}$ with $R_{3}$ generating $s u(2)_{R}$, while the remaining four pair of roots in $\mathcal{L}^{ \pm}$transform as a vector, i.e. in the $\operatorname{IRR}\left(\frac{1}{2}, \frac{1}{2}\right)$ of $s u(2)_{L} \oplus s u(2)_{R}$ as it is already implied by the three-graded decomposition given in (3.2). We note that these are complex vectors since $E_{ \pm\left(e^{i} \pm e^{j}\right)}^{\dagger}=$ $E_{\mp\left(e^{i} \pm e^{j}\right)}$.

In the oscillator basis introduced via (3.4), $\mathrm{SU}(2)_{L}$ and $\mathrm{SU}(2)_{R}$ generators can be built in the form

$$
L_{\alpha \beta}=a_{\alpha}^{\dagger} a_{\beta}-\frac{1}{2} \delta_{\alpha \beta} \hat{N}_{a}, \quad R_{\alpha \beta}=b_{\alpha}^{\dagger} b_{\beta}-\frac{1}{2} \delta_{\alpha \beta} \hat{N}_{b},
$$

while $\mathcal{L}^{+}$and $\mathcal{L}^{-}$are spanned by $a_{i}^{\dagger} b_{j}^{\dagger}$ and $a_{i} b_{j}$, respectively. The correspondence between the roots $E_{ \pm\left(e^{i} \pm e^{j}\right)}$ and $a_{i}^{\dagger} b_{j}^{\dagger}$ and $a_{i} b_{j}$, can be given explicitly as

$$
\begin{array}{rlrl}
a_{1}^{\dagger} b_{1}^{\dagger} & \equiv E_{e^{1}+e^{3}}, & & a_{1} b_{1} \equiv E_{-\left(e^{1}+e^{3}\right)}, \\
a_{1}^{\dagger} b_{2}^{\dagger} \equiv E_{e^{2}+e^{3}}, & & a_{1} b_{2} \equiv E_{-\left(e^{2}+e^{3}\right)}, \\
a_{2}^{\dagger} b_{1}^{\dagger} \equiv E_{-\left(e^{2}-e^{3}\right)}, & a_{2} b_{1} \equiv E_{e^{2}-e^{3}}, \\
a_{2}^{\dagger} b_{2}^{\dagger} \equiv E_{-\left(e^{1}-e^{3}\right)}, & a_{2} b_{2} \equiv E_{e^{1}-e^{3}} .
\end{array}
$$

Using a four component spinor of the form [38-40]

$$
\psi=\left(\begin{array}{c}
a_{1}^{\dagger} \\
a_{2}^{\dagger} \\
b_{1} \\
b_{2}
\end{array}\right), \quad \bar{\psi}=\psi^{\dagger} \Gamma^{6}=\left(-a_{1},-a_{2}, b_{1}^{\dagger}, b_{2}^{\dagger}\right),
$$

a Schwinger-type realization of the $s o(4,2)$ algebra is provided by

$$
M_{\mu \nu}=\bar{\psi} \Sigma_{\mu \nu}^{+} \psi
$$

where $\Sigma_{\mu \nu}^{+}$is the Fundamental four dimensional spinor IRR of $s o(4,2)$, whose relation to $\Gamma$ matrices of appropriate signature and dimension can be found in [38, 39]. Eq. (B.4) gives a unitary representation of $s o(4,2) \equiv s u(2,2)$ on the Fock space $\mathcal{F}$ generated by the action of $a_{\alpha}^{\dagger} b_{\alpha}^{\dagger}$ on the vacuum state with unit conformal energy. $\mathcal{F}$ partitions into direct sums of infinite number of unitary irreducible representations in two inequivalent ways as given in (3.6).

If we work with the doubleton corresponds to the upper sign in (3.7), using (B.2) explicit form of the action of the roots on the states $\left|h_{3}, J_{L}, J_{R}, m_{L}, m_{R}\right\rangle$ take the form

$$
\begin{aligned}
E_{ \pm\left(e^{1}+e^{2}\right)}\left|h_{3}, m_{L}, m_{R}\right\rangle= & \sqrt{\left(\frac{1}{2} h_{3}+\frac{1}{4} k \pm m_{L}+\frac{1}{2}\right)\left(\frac{1}{2} h_{3}+\frac{1}{4} k \mp m_{L}-\frac{1}{2}\right)} \\
& \times\left|h_{3}, m_{L} \pm 1, m_{R}\right\rangle, \\
E_{ \pm\left(e^{1}-e^{2}\right)}\left|h_{3}, m_{L}, m_{R}\right\rangle= & \sqrt{\left(\frac{1}{2} h_{3}-\frac{1}{4} k \pm m_{R}+\frac{1}{2}\right)\left(\frac{1}{2} h_{3}-\frac{1}{4} k \mp m_{R}-\frac{1}{2}\right)} \\
& \times\left|h_{3}, m_{L}, m_{R} \pm 1\right\rangle,
\end{aligned}
$$




$$
\begin{aligned}
E_{ \pm\left(e^{1}+e^{3}\right)}\left|h_{3}, m_{L}, m_{R}\right\rangle= & \sqrt{\left(\frac{1}{2} h_{3}+\frac{1}{4} k+m_{L} \pm \frac{1}{2}\right)\left(\frac{1}{2} h_{3}-\frac{1}{4} k+m_{R} \pm \frac{1}{2}\right)} \\
& \times\left|h_{3} \pm 1, m_{L} \pm \frac{1}{2}, m_{R} \pm \frac{1}{2}\right\rangle, \\
E_{ \pm\left(e^{2}+e^{3}\right)}\left|h_{3}, m_{L}, m_{R}\right\rangle= & \sqrt{\left(\frac{1}{2} h_{3}+\frac{1}{4} k+m_{L} \pm \frac{1}{2}\right)\left(\frac{1}{2} h_{3}-\frac{1}{4} k-m_{R} \pm \frac{1}{2}\right)} \\
& \times\left|h_{3} \pm 1, m_{L} \pm \frac{1}{2}, m_{R} \mp \frac{1}{2}\right\rangle, \\
E_{\mp\left(e^{2}-e^{3}\right)}\left|h_{3}, m_{L}, m_{R}\right\rangle= & \sqrt{\left(\frac{1}{2} h_{3}+\frac{1}{4} k-m_{L} \pm \frac{1}{2}\right)\left(\frac{1}{2} h_{3}-\frac{1}{4} k+m_{R} \pm \frac{1}{2}\right)} \\
& \times\left|h_{3} \pm 1, m_{L} \mp \frac{1}{2}, m_{R} \pm \frac{1}{2}\right\rangle, \\
E_{\mp\left(e^{1}-e^{3}\right)}\left|h_{3}, m_{L}, m_{R}\right\rangle= & \sqrt{\left(\frac{1}{2} h_{3}+\frac{1}{4} k-m_{L} \pm \frac{1}{2}\right)\left(\frac{1}{2} h_{3}-\frac{1}{4} k-m_{R} \pm \frac{1}{2}\right)} \\
& \times\left|h_{3} \pm 1, m_{L} \mp \frac{1}{2}, m_{R} \mp \frac{1}{2}\right\rangle .
\end{aligned}
$$

As for the doubleton with the lower sign in (3.7), we can simply take $k \rightarrow-k$ in the coefficients provided above.

Open Access. This article is distributed under the terms of the Creative Commons Attribution License (CC-BY 4.0), which permits any use, distribution and reproduction in any medium, provided the original author(s) and source are credited.

\section{References}

[1] S.M. Haaker, F.A. Bais and K. Schoutens, Noncompact Dynamical Symmetry of a Spin-orbit Coupled Oscillator, Phys. Rev. A 89 (2014) 032105.

[2] C.L. Kane and E.J. Mele, $Z_{2}$ Topological Order and the Quantum Spin Hall Effect, Phys. Rev. Lett. 95 (2005) 146802 [cond-mat/0506581] [INSPIRE].

[3] B.A. Bernevig and S.-C. Zhang, Quantum Spin Hall Effect, Phys. Rev. Lett. 96 (2006) 106802 [cond-mat/0504147] [INSPIRE].

[4] X.-L. Qi and S.-C. Zhang, The quantum spin Hall effect and topological insulators, Phys. Today 63 (2010) 33 [arXiv: 1001.1602].

[5] M.Z. Hasan and C.L. Kane, Topological Insulators, Rev. Mod. Phys. 82 (2010) 3045 [arXiv: 1002.3895] [INSPIRE].

[6] X.L. Qi and S.C. Zhang, Topological insulators and superconductors, Rev. Mod. Phys. 83 (2011) 1057 [arXiv: 1008. 2026] [INSPIRE].

[7] A. Schnyder, S. Ryu, A. Furusaki and A. Ludwig, Classification of topological insulators and superconductors in three spatial dimensions, Phys. Rev. B 78 (2008) 195125 [arXiv: 0803.2786] [INSPIRE]. 
[8] A. Kitaev, Periodic table for topological insulators and superconductors, in Proceedings of the L.D. Landau Memorial Conference Advances in Theoretical Physics, Chernogolovka Russia (2008) [arXiv: 0901.2686].

[9] S. Ryu, A.P. Schnyder, A. Furusaki and A.W.W. Ludwig, Topological insulators and superconductors: ten-fold way and dimensional hierarchy, New J. Phys. 12 (2010) 065010 [arXiv: 0912.2157].

[10] X.-L. Qi, T. Hughes and S.-C. Zhang, Topological Field Theory of Time-Reversal Invariant Insulators, Phys. Rev. B 78 (2008) 195424 [arXiv:0802.3537] [INSPIRE].

[11] B. Estienne, N. Regnault and B.A. Bernevig, D-Algebra Structure of Topological Insulators, Phys. Rev. B 86 (2012) 241104 [arXiv:1202.5543] [INSPIRE].

[12] T. Neupert, L. Santos, S. Ryu, C. Chamon and C. Mudry, Noncommutative geometry for three-dimensional topological insulators, Phys. Rev. B 86 (2012) 035125 [arXiv:1202.5188] [INSPIRE].

[13] S. Ryu and T. Takayanagi, Topological Insulators and Superconductors from D-branes, Phys. Lett. B 693 (2010) 175 [arXiv:1001.0763] [INSPIRE].

[14] A. Furusaki, N. Nagaosa, K. Nomura, S. Ryu and T. Takayanagi, Electromagnetic and thermal responses in topological matter: Topological terms, quantum anomalies and D-branes, C.R. Phys. 14 (2013) 871 [arXiv:1211.0533] [InSPIRE].

[15] Y. Li and C. Wu, High-Dimensional Topological Insulators with Quaternionic Analytic Landau Levels, Phys. Rev. Lett. 110 (2013) 216802 [arXiv:1103.5422].

[16] Y. Li, S.-C. Zhang and C. Wu, Topological insulators with SU(2) Landau levels, Phys. Rev. Lett. 111 (2013) 186803 [arXiv:1208.1562] [INSPIRE].

[17] Y. Li, K. Intriligator, Y. Yu and C. Wu, Isotropic Landau levels of Dirac fermions in high dimensions, Phys. Rev. B 85 (2012) 085132 [arXiv:1108.5650] [INSPIRE].

[18] M. Asorey, A.P. Balachandran and J.M. Pérez-Pardo, Edge States: Topological Insulators, Superconductors and QCD Chiral Bags, JHEP 12 (2013) 073 [arXiv:1308.5635] [INSPIRE].

[19] K. von Klitzing, G. Dorda and M. Pepper, New method for high accuracy determination of the fine structure constant based on quantized Hall resistance, Phys. Rev. Lett. 45 (1980) 494 [INSPIRE].

[20] D.C. Tsui, H.L. Stormer and A.C. Gossard, Two-dimensional magnetotransport in the extreme quantum limit, Phys. Rev. Lett. 48 (1982) 1559 [INSPIRE].

[21] D.J. Thouless, M. Kohmoto, M.P. Nightingale and M. den Nijs, Quantized Hall Conductance in a Two-Dimensional Periodic Potential, Phys. Rev. Lett. 49 (1982) 405 [InSPIRE].

[22] M. Kohmoto, Topological invariant and the quantization of the Hall conductance, Annals Phys. 160 (1985) 343.

[23] R.B. Laughlin, Anomalous quantum Hall effect: An Incompressible quantum fluid with fractionallycharged excitations, Phys. Rev. Lett. 50 (1983) 1395 [INSPIRE].

[24] S.C. Zhang, T.H. Hansson and S. Kivelson, An effective field theory model for the fractional quantum hall effect, Phys. Rev. Lett. 62 (1988) 82 [INSPIRE].

[25] S.-C. Zhang, The Chern-Simons-Landau-Ginzburg theory of the fractional quantum Hall effect, Int. J. Mod. Phys. B 6 (1992) 25 [INSPIRE].

[26] F.D.M. Haldane, Model for a Quantum Hall Effect without Landau Levels: Condensed-Matter Realization of the 'Parity Anomaly', Phys. Rev. Lett. 61 (1988) 2015 [InSPIRE]. 
[27] S.-C. Zhang and J.-p. Hu, A Four-dimensional generalization of the quantum Hall effect, Science 294 (2001) 823 [cond-mat/0110572] [INSPIRE].

[28] D. Karabali and V.P. Nair, Quantum Hall effect in higher dimensions, Nucl. Phys. B 641 (2002) 533 [hep-th/0203264] [InSPIRE].

[29] B.A. Bernevig, J.-p. Hu, N. Toumbas and S.-C. Zhang, The Eight-dimensional quantum Hall effect and the octonions, Phys. Rev. Lett. 91 (2003) 236803 [cond-mat/0306045] [inSPIRE].

[30] K. Hasebe and Y. Kimura, Dimensional hierarchy in quantum Hall effects on fuzzy spheres, Phys. Lett. B 602 (2004) 255 [hep-th/0310274] [INSPIRE].

[31] Ü.H. Coşkun, S. Kürkçüoğlu and G.C. Toga, Quantum Hall Effect on Odd Spheres, Phys. Rev. D 95 (2017) 065021 [arXiv: 1612.03855] [InSPIRE].

[32] P.A.M. Dirac, A Remarkable representation of the $3+2$ de Sitter group, J. Math. Phys. 4 (1963) 901 [InSPIRE].

[33] M. Flato and C. Fronsdal, One massless particle equals two Dirac singletons, Lett. Math. Phys. 2 (1978) 421.

[34] Z.F. Ezawa, Quantum Hall Effects, third edition, World Scientific, Singapore (2013).

[35] A. Cappelli, C.A. Trugenberger and G.R. Zemba, Infinite symmetry in the quantum Hall effect, Nucl. Phys. B 396 (1993) 465 [hep-th/9206027] [InSPIRE].

[36] S. Iso, D. Karabali and B. Sakita, Fermions in the lowest Landau level: Bosonization, W infinity algebra, droplets, chiral bosons, Phys. Lett. B 296 (1992) 143 [hep-th/9209003] [INSPIRE].

[37] J. Martinez and M. Stone, Current operators in the lowest Landau level, Int. J. Mod. Phys. B 7 (1993) 4389 [InSPIRE].

[38] K. Govil and M. Günaydin, Deformed Twistors and Higher Spin Conformal (Super-)Algebras in Four Dimensions, JHEP 03 (2015) 026 [arXiv: 1312.2907] [INSPIRE].

[39] K. Govil and M. Günaydin, Deformed Twistors and Higher Spin Conformal (Super-)Algebras in Six Dimensions, JHEP 07 (2014) 004 [arXiv: 1401.6930] [INSPIRE].

[40] M. Sperling and H.C. Steinacker, The fuzzy 4-hyperboloid $H_{n}^{4}$ and higher-spin in Yang-Mills matrix models, Nucl. Phys. B 941 (2019) 680 [arXiv:1806. 05907] [INSPIRE].

[41] S. Fernando and M. Günaydin, Massless conformal fields, $A d S_{d+1} / C F T_{d}$ higher spin algebras and their deformations, Nucl. Phys. B 904 (2016) 494 [arXiv:1511.02167] [INSPIRE].

[42] M. Günaydin, Quasiconformal Group Approach to Higher Spin Algebras, their Deformations and Supersymmetric Extensions, in Proceedings of International Workshop on Higher Spin Gauge Theories, Singapore (2015), pg. 159 [arXiv: 1603. 02359] [INSPIRE].

[43] A. Böhm, Quantum Mechanics, Texts and Monographs in Physics, Springer, New York U.S.A. (1979).

[44] A.P. Balachandran, G. Immirzi, J. Lee and P. Prešnajder, Dirac operators on coset spaces, J. Math. Phys. 44 (2003) 4713 [hep-th/0210297] [INSPIRE].

[45] A. Perelomov, Generalized Coherent States and their Applications, Springer-Verlag, Heidelberg Germany (1986).

[46] J. Fuchs and C. Schweigert, Symmetries, Lie Algebras and Representations: A Graduate Course for Physicists, Cambridge University Press, Cambridge U.K. (2003). 